PDFlib PLOP: PDF Linearization, Optimization, Protection

Page inserted by evaluation version www.pdflib.com - sales@pdflib.com 


\title{
Massive Black Holes in Nearby Galaxies
}

\author{
DOUGLAS RICHSTONE
}

\author{
Department of Astronomy \\ University of Michigan \\ Ann Arbor, Michigan 48109 \\ INTRODUCTION
}

Very soon after the recognition of large redshifts of quasars, Salpeter ${ }^{1}$ proposed that the energy source was gravitational. Lynden-Bell ${ }^{2}$ has since shown that the luminosity, variability and probable lifetimes of these objects indicate that "gravity power" outshines nuclear power, under a very inclusive set of assumptions. Rees ${ }^{3}$ has argued that a wide variety of relativistic alternatives for the gravitational potential well (for example, a relativistic star cluster) are likely to evolve to massive black holes. Many of the theoretical issues and observational inferences of this very productive paradigm are reviewed by Rees and by Bregman ${ }^{4}$.

Although these sorts of arguments defend the success of this model, it is disturbing that no calculation of the evolution of a nonrelativistic object to a massive black hole exists, and the evidence, to date, for the existence of massive black holes in quasars, is circumstantial.

In my view, one of the most elegant consistency checks of the black hole accretion model for quasars is due to P.J. Young, W. Sargent, and their collaborators ${ }^{5,6}$. In their view, the fact of rapid evolution of the quasar number density demands the presence of dead quasars in the present universe. The presence of moderate redshift quasars in galaxies ${ }^{7,8}$ and the hypothesis that quasars are powered by accretion onto massive black holes then require the presence of such objects in some galactic nuclei at present, unless they have been flung out (a possibility that has not been investigated). This report is focused on results of various searches of nearby galaxies for candidates for massive black holes that might be expected under this theory.

\section{EXPECTED FREQUENCY AND MASSES OF DEAD QUASARS}

We may estimate the density of the mass $\rho$ of the total luminosity released by quasars during the history of the universe from the observed conditional distribution $\Phi(L \mid z)$ of luminosity $L$, given redshift $z$, from

$$
\rho c^{2}=\int_{0}^{\infty} \int_{0}^{\infty} \mathrm{L} \Phi(\mathrm{L} \mid \mathrm{z}) \mathrm{dL}(\mathrm{dt} / \mathrm{d} \mathrm{z}) \mathrm{d} z
$$


which leads to a mass density of relic black holes of

$$
\rho=220,000 / \epsilon_{0.1} M_{\odot} \mathrm{Mpc}^{-3}
$$

where $\epsilon_{0.1}$ is the efficiency of mass - energy conversion by the accretion process, normalized to $10 \%$. The estimate above (the best currently available) is from Chokshi and Turner ${ }^{9}$, slightly improving the method of Soltan ${ }^{10}$. This estimate includes a rather uncertain bolometric correction. It seems rather more likely to be an underestimate than an overestimate. Somewhat remarkably, the density of quasar relics computed in this fashion is completely independent of the Hubble constant and of $\Omega_{0}$. The mass contained in a sphere of radius $16 \mathrm{Mpc}$ (the distance to M87) according to eqn 2 is $M_{16} \approx 3 \times 10^{9} M_{\odot} \epsilon_{0.1}$.

Estimates of the masses of quasar engines are model dependent, but, using the Eddington limit as a guide, we may expect that relic black hole masses are at least $M_{\bullet} \approx 10^{8} M_{\odot} \times \mathrm{L} / 10^{46} \mathrm{ergs} / \mathrm{sec}$.

\section{EVIDENCE FROM DYNAMICAL SEARCHES}

The least ambiguous data we have at present comes from inferences drawn from the stellar dynamics of galactic centers. In several cases, spectra of the central regions of nearby galaxies reveal the presence of a very large stellar velocity dispersion or rotation or both. The interpretation of these observed velocity moments (which are projected on the line of sight and integrated through the galaxy) are complicated by the possibility of varying anisotropy in the velocity distribution function of the stellar fluid. Techniques to deal with this difficulty by surveying configurations that are solutions of the collisionless Boltzmann equation have been developed ${ }^{11}$. In a number of cases there is clear evidence for increasing $M / L$ in the central region of a galaxy. In all cases this effect can be produced by a constant $M / L$ stellar population and a point mass at the galactic center. At this point a number of galaxies have been surveyed fairly carefully to study the consistency of the frequency of these objects with the predicted frequency of quasar relics. The table below gives my view of the current status of these surveys.

Table 1 Survey Results for Massive Black Hole Candidates

\begin{tabular}{llll}
\hline Galaxy & $M_{\odot} / 10^{8} M_{\odot}$ & Notes & References \\
\hline M33 & $\leq 5 \times 10^{-4}$ & upper limit & 12 \\
M31 & $0.1-0.7$ & & $13,14,15$ \\
M32 & $0.007-0.08$ & & $13,15,16$ \\
NGC 4594 & $\sim 10$ & & 17 \\
NGC 2613 & $\leq 30$ & limit; LINER/AGN & 18 \\
NGC 4699 & $\leq 30$ & limit; LINER/AGN & 18
\end{tabular}




$\begin{array}{llll}\text { NGC } 5747 & \leq 10 & \text { limit; LINER/AGN } & 18 \\ \text { NGC 7331 } & \leq 10 & \text { limit; LINER } & 18 \\ \text { M87 } & \leq 30 & & 19,20 \\ \text { NGC 3115 } & 1-10 & & 21\end{array}$

It is clear that the number density of massive black hole candidates is not wildly inconsistent with the expected density of dead quasars. Furthermore, a plot of the contents of Table 1 would reveal a strong correlation with either bulge (or elliptical galaxy) mass ${ }^{22}$ or with distance. This correlation is certainly partly due to the combined effect of selection and the distance dependence of the minimum discoverable mass ${ }^{23}$. The ability to discover a $M / L$ gradient depends on measuring $M \propto v^{2} r$. The Local Group contains only small bulge galaxies and only posesses low mass massive black hole candidates. At Virgo distances, a mass of $10^{9} M_{\odot}$ is necessary (but not sufficient) for detection. The possibility remains that there is a correlation

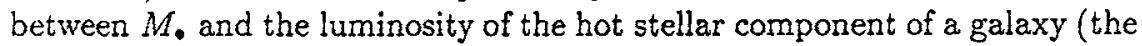
total luminosity of an elliptical, or the luminosity of the bulge of a spiral).

\section{EVIDENCE FROM IMAGES ALONE}

Space Telescope (ST) data has yielded new information on the distribution of starlight near the centers of a few galaxies. These results are particularly interesting for M32 and M87. In each case ${ }^{24,25}$, there is evidence that the starlight distribution does not exhibit a constant density core. Rather, there is a mild divergence in intensity, but not luminosity, at the center of the galaxy. A constant density core is expected if the galaxies form by violent relaxation ${ }^{26}$. Mild cusps in the light distribution are to be expected from adiabatic pulling of stellar orbits due to growth in central black hole mass ${ }^{27,28}$, but might also be expected from gas flow and secondary star formation, or possibly by accretion of globular clusters ${ }^{29}$. If one rejects a massive black hole in these galaxies, one is forced to account for the mild cusps that are observed, and the beautiful agreement between the ST starlight profile for M87 and reference 28 must be seen to be an unfortunately misleading accident. In my view, the ST data do not yet much strengthen the case for massive black holes in these objects. Further theory and observation with existing instruments should be illuminating.

A different kind of evidence from ST appears in the form of a widely distributed photograph of NGC 4261 by Jaffe et al. , showing what appears to be an edge brightened disk or toroid, with a bright central region, and with a position angle roughly normal to the galaxy's radio jets. On one hand, the geometrical structure and jet orientation are consistent with popular prejudice. On the other, the spatial resolution is $10^{2}$ worse than the scale of a possible thermal accretion disk, and $10^{5}$ worse than the scale on which a relativistic jet would be collimated. 


\section{POTENTIAL PROBLEMS}

The greatest weakness in this discussion is that we are unable to probe even the nearby galaxies with sufficient resolution to observe stars with $v \sim c$, which would be a fairly unambiguous indicator of massive black holes. Thus we are unable to rule out alternate models of $M / L$ gradients at the centers of galaxies.

Other interesting questions concern the relation of putative nuclear massive black holes to their surroundings and may be phrased, following J. Goodman (unpublished), as, why are they nuclear, why are they massive, and why are they black? The first two questions really address the question of formation and evolution in relation to the nucleus of the galaxy. Did accretion pull in the nuclear cusp in an otherwise smooth distribution or is the black hole a consequence of core collapse of a dynamically evolved nucleus?

The third question is troubling. It is a little surprising that these objects are as dark as they are. The presence of massive black holes in galactic nuclei predicts the occurence of stellar breakup flashes ${ }^{30,31,32}$, of total energy $\sim \epsilon M_{\odot} c^{2}=\epsilon 10^{54} \mathrm{ergs}$, but uncertain duration, bolometric correction and frequency. These flashes result from the circularization and accretion of tidal debris of stars scattered from safe orbits in the galactic nucleus to orbits with pericenters internal to the star's Roche limit. In M31, the expected frequency is about $10^{4}$ years. A search for these flashes has the potential to contribute an important new perspective to this problem.

\section{SUMMARY}

There are now a handful of cases in which $M / L$ rises sharply at the center of a galaxy as determined directly from observations of the dynamics of stars in the galaxy. A massive nuclear black hole is a likely cause of this phenonenon. In no case can one unambiguously rule out other possible explanations, but M31 might well present such a case soon (see reference 15). Space Telescope starlight profiles of galaxies are surprisingly cuspy in a few cases. The interpretation of this is interesting but ambiguous. If nuclear massive black holes are common we should observe occasional stellar breakup thashes of great energy.

\section{REFERENCES}

1. Salpeter, E. E. $1964 A p J$ 140: $796-800$.

2. Lynden-Bell, D. 1978 Physica Scripta 17: 185 - 191.

3. Rees, M. J. 1984 Ann Rev Astron and Astroph 22: 507 - 536.

4. Bregman, J. N. 1990 Astron Astroph Review 2: 125 - 165.

5. Young, P. J., Westphal, J. A., Kristian, J., Wilson, C. P. and Landauer, F. P. ApJ 221, $721-730$. 
6. Sargent, W. L. W., Young, P. J., Boksenberg, A., Shortridge, K., Lynds, C. R., and Hartwick, F. D. A. Whelan, J. A. J. ApJ 221, $731-744$.

7. Boroson, T. A. and Oke, J. B. 1984 ApJ 281, 535 - 544.

8. Smith, E., Heckman, T., Bothun, G., Romanishin, W., and Balik, B 1986 ApJ 306, $64-89$.

9. Chokshi, A. and Turner, E. L. 1992 MNRAS 259, $421-424$.

10. Soltan, A. 1982 MNRAS 200, 115- 122.

11. Richstone, D., and Tremaine, S. 1988 ApJ 327, $82-88$.

12. Kormendy, J. and McClure, R. D. $1993 A J$ in press.

13. Dressler, A, and Richstone, D. 1988 ApJ 324, 701 - 713.

14. Kormendy, J. $1988 A p J 325.128-141$

15. Richstone, D., Bower, G. and Dressler, A. 1990 ApJ 353, $118-122$.

16. Tonry, J. L. 1984 ApJ (Letters) 283, L27 - 30.

17. Kormendy, J. 1988 ApJ 335, $40-56$.

18. Bower, G. A., Richstone, D. O., Bothun, G. D., and Heckman, T. M. 1993 ApJ 402, 76 - 94.

19. Richstone, D. and Tremaine, S. $1985 A p J$ 296, $370-378$.

20. Dressler, A. and Richstone, D. $1990 A p J 348,120-126$.

21. Kormendy, J. and Richstone, D. 1992 ApJ 393, 559 - 578.

22. Kormendy, J. 1993 in The Nearest Active Galaxies ed. Beckman, Netzer and Colina.

23. Richstone, D. 1988 in Supermassive Black Holes ed. M. Kafatos, p. 87.

24. Lauer, T. et al. 1992 AJ 104, $552-562$.

25. Lauer, T. et al. 1992 AJ 103, $703-710$.

26. Tremaine, S. 1987 in Structure and Dynamics of Elliptical Galaxies ed. $T$. de Zeeuw.

27. Young, P. 1980 ApJ 242, 1232 - 1237.

28. Lee, M. H. and Goodman, J 1989 ApJ 343, $594-601$.

29. Tremaine, S., Oskriker, J. P. and Spitzer, L. 1975 ApJ 196, 407 -411.

30. Goodman, J., and Lee, H. M. $1989 A p J$ 337, 84 - 90.

31. Rees, M. J. 1988 Nature $353,523-526$.

32. Canizzo, J. K., Lee, H. M. and Goodman, J. 1990 ApJ 351, 38 - 46. 